\title{
CONCORDANCIA ENTRE LA FACULTAD ACADÉMICA A LA QUE PERTENECE EL ALUMNO CON LOS INTERESES VOCACIONALES Y CON LOS ESTILOS ATRIBUCIONALES DE LAS CAUSAS QUE DETERMINARON EL INGRESO A LA UNMSM EN EL CONCURSO DE ADMISIÓN 2000
}

\author{
Luis Alberto Vicuña Peri \\ Mildred Paredes Tarazona \\ Héctor Hernandez Valz \\ Rolando Solís Narro
}

\begin{abstract}
RESUMEN
La presente investigación trata sobre la concordancia entre los intereses vocacionales y la Escuela Académico Profesional a la que ingresó el estudiante en el proceso de selección del año 2000 .

La evaluación de los intereses se realizó mediante un cuestionario elaborado para el presente estudio. El segundo instrumento utilizado evaluó los estilos atribucionales. Estas pruebas se aplicaron a una muestra aleatoria conformada por 20 escuelas académicas de las diecinueve Facultades con las que cuenta la Universidad Nacional Mayor de San Marcos requiriendo 698 examinados con un nivel de confianza del $95 \%$ y con un error de estimación del 0.05 .

Del total de examinados el 22\% comunican su deseo de cambiarse de especialidad, sin diferencia por el género. Las razones de quienes dicen que se cambiarán son las siguientes: porque no es su vocación, porque no hay trabajo, y porque la enseñanza es mala. La intención del traslado se dirige hacia medicina humana, ingeniería de sistemas y derecho.

El estilo atribucional tiende hacia el pesimismo principalmente por la tendencia a autoresponsabilizarse de manera muy estable de acontecimientos que no dependen de ellos directamente, sin embargo es manejable. En suma, los traslados son promovidos por razones de vocación, y el ingreso a una especialidad diferente corresponde a su plan de vida sin que influyan ni el género ni la especialidad a la que se ingresó, hallazgo que hace necesario implementar el servicio permanente de consejería y orientación vocacional.
\end{abstract}

Palabras clave: Expectativa, percepción, estilo atribucional, vocación.

\begin{abstract}
The present investigation tries on the agreement between the vocational interests and the School Professional Academic to which the student entered in the process of selection of the year2000.

The evaluation of the interests was carried out by means of a questionnaire elaborated for the present study. The second used instrument evaluated the attributional styles. These tests were applied to an aleatory sample conformed by 20 academic schools of the nineteen Abilities with those that it counts the National University bigger than San Marcos requiring 698 examined with a level of trust of $95 \%$ and with an error of estimate of the 0.05 .

Of the total of having examined $22 \%$ communicate their desire to be changed specialty, without difference for the gender. The reasons of those who say that they will be changed they are the following ones: because it is not their vocation, because there is not work, and because the teaching is bad. The intention of the transfer goes toward human medicine, engineering of systems and right.

Attributional style tends towards pessimism mainly by the tendency to self-responsibility very stable so that events do not depend on them directly, yet it is manageable. In sum, the transfers are promoted by vocation reasons, and the en trance to a different specialty it corresponds to their plan of life without they influence neither the gender neither the specialty to which was entered, discovery that makes necessary to implement the permanent service of counselling and vocational orientation.
\end{abstract}

Key Words: Expectation, perception, attributional style, vocation. 
Es preocupante el resultado del análisis de datos provenientes de los cuadros de ingresantes y matriculados por años, publicados por la Oficina General de Investigación y Planificación (Boletín Nro. 6. Año. 1997) y de datos proporcionados por quince Escuelas Académicos Profesionales que tuvieron a bien proporcionarnos la información necesaria. Dicho resultado consiste fundamentalmente en que entre el número de postulantes que ingresaron a la Universidad Nacional Mayor de San Marcos, de los años 1990 a 1997, y el número de matriculados en los años subsiguientes, (donde teóricamente se debe esperar correspondencia numérica siempre que hubiera una trayectoria longitudinal de desempeño académico); tiene como resultados las siguientes cifras: alrededor del $29 \%$ de los alumnos, que ingresaron no se matriculan tres años después y cerca del $32 \%$ no concluyen sus estudios con su grupo de referencia. Aunque estos resultados son parciales en relación con el total de escuelas, se puede estimar que es muy probable que los porcentajes de deserción sean aún mayores llegando, a comprometer a todas las Escuelas Académico Profesionales con mayor incidencia en unas que en otras.

Se puede enumerar un conjunto muy amplio y diverso de las posibles causas de la disminución progresiva de los estudiantes que se matriculan en el periodo académico correspondiente, las que pueden agruparse en factores económicos, salud, matrimonios inesperados, y psicológicos. En este último aspecto nos interesa explorar con que expectativas y percepciones vocacionales ingresan los estudiantes, respecto a su Escuela Académico Profesional, puesto que las discrepancias entre lo percibido con lo hallado concretamente, son fuentes que generan en el individuo sentimientos de frustración.

Estos sentimientos no se atenúan con el mero conocimiento de lo que la profesión ofrece, por lo contrario tienden a fortalecerse, gravitando en favor del descontento que va asociado al desapego con el estudio, aumentando así, la probabilidad de la desaprobación o repitencia de las asignaturas, y con ello el distanciamiento con la actividad académica. De este recorrido solo queda como síntesis, la configuración de un estudiante con el perfil de candidato para la deserción o para el desfase en una o más promociones de egreso.

$\mathrm{Si}$ a las expectativas y percepciones de la profesión le agregamos los estilos atribucionales de las causas que determinaron el ingreso a la universidad podremos identificar si la percepción del ingresante es compatible con la habilidad y el esfuerzo o por lo contrario con la dificultad y la suerte. El desconocimiento de estos factores psicológicos en nuestros estudiantes llevará a que la tasa de deserciones continúe con perjuicios muy grandes, no sólo de naturaleza económica sino lo que resulta más importante, la perdida de los recursos humanos y lo que esto significa en el plano del desarrollo individual, la marginación hacia el despliegue de sus potencialidades.

El presente estudio está enmarcado dentro de la Psicología Educativa, que es una de las áreas de desarrollo del Instituto de Investigaciones Psicológicas, y su ejecución nos permitirá identificar si las expectativas, las percepciones vocacionales y los estilos atribucionales guardan concordancia con la facultad a la que se ingresó. Del examen de los hallazgos se podrá proponer a las instancias pertinentes la incorporación de criterios vocacionales para la admisión de los postulantes y desarrollar los programas pertinentes orientados a optimizar los estilos atribucionales del pesimismo hacia el optimismo donde la habilidad y el esfuerzo primen sobre la dificultad y la suerte.

Según lo expuesto cuando trabajamos con una muestra de estudiantes de las diferentes Escuelas Académico Profesional de la Universidad Nacional Mayor de San Marcos, ingresantes en el concurso de admisión 2 000, se quiere despejar las siguientes interrogantes: 
- ¿Los estudiantes que declaran su deseo por cambiarse de especialidad, difieren en sus expectativas y percepciones hacia la profesión elegida así como en sus estilos atribucionales respecto de sus pares que contrariamente declaran haber alcanzado su meta?

- ¿El género y la facultad a la que se ingresó tienen algún papel significativo al respecto?

Las expectativas se refieren a los motivos como elementos impulsores (Tolman, en Madsen 1967) que actúan como señales para orientar selectivamente la conducta. Las expectativas se constituyen en el hombre en íntima interdependencia con su historia personal y la influencia que el entorno ejerce alrededor del status qua. El individuo en el presente, se proyecta hacia el futuro, de dos formas no necesariamente excluyentes. La primera forma es pasiva aquí, el sujeto espera que las cosas ocurran de conformidad a sus deseos e imaginaciones. En la segunda forma se proyecta en lo que tendrá que hacer, en qué momento y de que manera. Si predomina la primera forma es muy probable que el sujeto tenga muy poca resistencia a la frustración. De predominar la segunda el sujeto será muy emprendedor siempre que juzgue que es el momento apropiado, aquí puede estar el problema, si el sujeto es vacilante posiblemente demore mucho para pasar a la acción, estos sujetos no se sienten frustrados, están convencidos que aún no han empezado.

La armonía entre la primera y segunda forma nos pondría ante un sujeto que ha logrado compatibilizar sus deseos con sus posibilidades basados en el conocimiento de sus capacidades actuales, estos sujetos se acomodan muy bien al estilo de vida productivo de Adler (Titze, 1985) y también a los sujetos con motivación de logro de McClellan, (McCllellan en Madsen, 1967).

Aplicando lo descrito a la situación de ingreso a la universidad, podremos encontramos con estudiantes que se ubican en cualquiera de las tres formas, siendo muy probable que las los traslados a otra especialidad, deserciones, repitencias y abandono temporales ocurran en las dos primeras formas. De ser así la información previa, clara y puntual podría tener algún efecto preventivo.

La percepción es el proceso psicológico mediante el cual el sujeto otorga significados a todo aquello que lo rodea y de lo que es consciente. La percepción nos permite organizar el mundo conceptualmente, (Hamlyn, 1981). En el presente estudio la percepción nos permitirá conocer lo que la universidad y la especialidad elegida significa para el estudiante. Los significados podrán ser favorables o por lo contrario cargados de contenidos desalentadores.

Combinando las expectativas armónicas con las percepciones favorables, el pronóstico hacia la autorrealización humana es positiva, en tanto que el pronóstico será reservado cuando ocurren combinaciones entre cualquiera de las dos primeras formas de expectativas con las percepciones desfavorables.

Explorar las expectativas y las percepciones hacia la especialidad a la que ingresaron los estudiantes universitarios es una forma de identificar si hay compatibilidad entre sus intereses vocacionales con la Escuela Académico Profesional a la que ingresaron, de haber compatibilidad debemos esperar permanencia hasta culminar exitosamente la profesión y lo contrario si la compatibilidad es forzada o ausente.

La vocación como la resultante de componentes motivacionales, aptitudinales y de personalidad se encargará de mantener firme la conducta del sujeto hacia la conquista de sus metas, sean estas profesionales, técnicas o de oficio. La vocación ha sido estudiada 
desde diferentes enfoques pero nos interesa mencionar dos: el enfoque evolutivo y el enfoque conductual cognitivo.

El enfoque evolutivo está basado en la teoría del desarrollo, su supuesto teórico afirma que lo vocacional tiene un carácter procesal y acompaña el desarrollo de la persona desde la preparación a través de la educación hasta el retiro de la vida laboral activa. Cada fase tiene elevado grado de irreversibilidad y concreta o actualiza una gama de conductas o potencialidades personales. La madurez vocacional está relacionada con el ajuste y compromiso personal.

El enfoque conductual-cognitivo asume los principios del conductismo y del cognitivismo respecto a las teorías del aprendizaje social, el procesamiento de la información, el pensamiento y la propositividad comportamental. El supuesto teórico contempla la conducta vocacional como multidimensional, condicionada por aprendizajes anteriores, interactuando elementos individuales con factores estimulares del medio sociocultural y, en conjunto sometida a regularidades. Lo vocacional es una manifestación global de la persona que orienta su vida a aspectos valiosos para ella y presumiblemente también para su entorno, lo que sitúa a esta acción comportamental en la cima de la socialización adulta.

El problema vocacional es idiosincrásico, vivido por una determinada persona en un determinado contexto y está abocado a la toma de decisiones en situación de incertidumbre parcial, por lo que el sujeto siente la necesidad de recibir ayuda especializada y asumir libremente sus propias responsabilidades.

En ambos enfoques observamos que la vocación tiene que ver con el ajuste y la realización personal asumiendo libremente sus propias responsabilidades.

La ocurrencia de lo descrito presupone un contexto con características idiosincrásicas homogéneas; sin embargo los estudiantes ingresante a la universidad, esta constituido por un grupo heterogéneo en su capacitación previa, en las expectativas de sus padres, en las expectativas de ascenso social, en el nivel socioeconómico-cultural, que hace de la labor formativa universitaria en los primeros años una etapa de transición entre la secundaria y la universidad propiamente dicha.

Esta situación, sobre la que no existen diagnósticos situacionales hasta ahora, nos lleva a focalizar e investigar uno de los puntos álgidos de la vida universitaria, nos referimos a la concordancia entre la especialidad a la que se ingresó con los intereses vocacionales expresados mediante las percepciones y expectativas.

Por otro lado, también hemos considerado muy importante averiguar el estilo atribucional de los ingresantes a la universidad para lo cual en los avances de la psicología por entender del control personal, según Peterson \& Stunkard (1992), el «Estilo Explicativo», es un constructo más comprehensivo y con mayor poder explicativo, que el de locus de control formulado por J. B. Rotter, y el de autoeficacia de A. Bandura. Aunque no los sustituya totalmente, comparte con estas y otras teorías del control personal las siguientes características (Peterson, 1992)

a) El control personal es uno de los modos más importantes, según el cual las personas se diferencian unas de otras.

b) El control personal reside en la transacción entre la persona y el mundo, no es sólo una disposición o sólo una característica del ambiente.

c) El control personal puede tomar la forma de la creencia en que uno puede afectar los resultados reales, elegir entre ellos, afrontar sus consecuencias, y/o entenderlas. 
d) En un ambiente retador, el control personal es deseable; éste da ánimo a las emociones, la conducta y el sistema fisiológico ante los retos.

e) El control personal puede ser frustrado por el fracaso y animado por el éxito, aunque esta no sea una relación uno a uno con los patrones de éxitos y fracasos del pasado.

Bernard Weiner y Albert Stunkard, en el artículo publicado en 1992, comparan las tres propuestas más conocidas que sobre control personal, se han venido desarrollando: locus de control, autoeficacia y estilo explicativo. Todas ellas tienen en común su impacto en las conductas futuras de las personas, aunque en diferentes niveles.

Cada una de las teorías mencionadas se ocupa de diferentes dimensiones y a diverso nivel de abstracción o generalización, siendo el locus de control un factor más específico, mientras que el estilo explicativo llega a ser de tal nivel de generalidad que incluso ha sido postulado como un rasgo constitutivo de la personalidad (Reeve, 1995).

La autoeficacia parece ser el constructo con más posibilidades de integración (Peterson \& Stunkard, 1992), mientras que el estilo explicativo también ha sido abundantemente respaldado por variedad de investigaciones que corroboran su poder predictivo no sólo en los ámbitos de la psicología de la salud sino también en el bienestar general como lo demuestran las investigaciones realizadas durante los últimos quince años (Peterson, Buchanan, \& Seligman, 1995).

Antes de revisar directamente los componentes del estilo explicativo o atribucional, veamos cómo se derivó ésta de la teoría atribucional de la motivación. Bernard Weiner es uno de los psicólogos que ha procurado integrar los hallazgos en esta área de investigación formulando una teoría atribucional de la motivación para el éxito o motivación de logro (Weiner, 1992). Para él, las personas suelen atribuir sus éxitos o fracasos a una de las siguientes cuatro posibles causas: habilidad, cantidad de esfuerzo, dificultad de la tarea y la suerte.

\begin{tabular}{|l|l|c|}
\cline { 2 - 3 } \multicolumn{1}{c|}{} & Interno & Externo \\
\hline $\begin{array}{l}\text { Estable } \\
\text { Inestable }\end{array}$ & $\begin{array}{l}\text { Habilidad } \\
\text { Esfuerzo }\end{array}$ & $\begin{array}{c}\text { Dificultad } \\
\text { Suerte }\end{array}$ \\
\hline
\end{tabular}

Estas cuatro fuentes de causalidad se integran en dos dimensiones: Estable Inestable, e Interno-Externo. La habilidad es entonces una causa interna al sujeto y relativamente estable en el tiempo, la cantidad de esfuerzo es el otro elemento de la dimensión interna pero que puede fácilmente variar de una situación a otra, es decir no es estable en el tiempo. Mientras que la dificultad de la tarea es un aspecto externo al sujeto y relativamente estable mientras que el factor suerte es externo y no constante o inestable pues no está bajo control de la persona y puede variar de una ocasión a otra.

En diversas publicaciones Weiner presenta resultados de investigaciones que evidencian la influencia de las atribuciones de causalidad en la persistencia, intensidad y la elección de determinadas conductas (Weiner, 1992).

A diferencia de la teoría de la expectativa por valor de Atkinson, en la teoría atribucional de Weiner las reacciones emocionales y las expectativas de éxito o fracaso son mediadas por la atribución hecha sobre la situación percibida antes de que el sujeto llegue a la acción (Weiner, 1992).

En el marco de la teoría atribucional de la motivación propuesta por Weiner, y tomando como punto de partida el modelo reformulado de la desesperanza aprendida, Peterson incluye además de las dos dimensiones de atribución consideradas por Weiner, 
una tercera que considera lo global o especifico del factor causal.

En el constructo denominado estilo explicativo o atribucional que confluyen por un lado la teoría de la atribución y por otro el de la desesperanza aprendida (Seligman, 1975). como un modo de explicar las diferencias individuales en las respuestas a eventos no controlables.

A partir de los estudios publicados por Seligman en 1975 y Abramson et al en 1978, Peterson (1991) identifica que el efecto de experiencias incontrolables y negativas producían en los sujetos la desesperanza aprendida pero en diversos modos. Por ello no se encontraban los mismos patrones que en los experimentos con animales. Tras varios años de investigación y una serie de formulaciones Peterson finalmente llegó a proponer como constitutivas del Estilo Explicativo las tres dimensiones causales mencionadas:

Interno-Externo, Estable-Inestable y Global - Específico.

El Estilo Explicativo es una variable cognitiva de personalidad que refleja el modo en que las personas explican habitualmente las causas de sus fracasos, da cuenta de la falta de motivación o desmoralización ante situaciones que demandan logros (Peterson, 1990).

Si una persona tiende a atribuir las razones de sus fracasos o eventos negativos a causas internas, entonces estará minando su propia autoestima, sentido de valía personal y percepción de su capacidad para controlar las situaciones.

Si a ello se aúna la tendencia a pensar que las causas de los infortunios sufridos van a permanecer más allá del día de hoy y de la situación específica presente, tendremos que la persona estará atribuyendo su condición a factores estables y globales.

La conjunción de una explicación interna, estable y global, es la que mayor pesimismo y desmoralización producirá en el individuo y es denominada por ello estilo explicativo pesimista.

Se ha encontrado que el estilo explicativo pesimista está asociado a la depresión e incluso a la pérdida de la salud físico (Peterson, 1992, 450), pero además de ellos Peterson cita los siguientes resultados como consecuentes de un estilo explicativo pesimista:

a) Depresión (Sweeney, Anderson, \& Bailey, 1986)

b) Bajas calificaciones en la escuela (Peterson \& Barret, 1987)

c) Bajo desempeño laboral (Seligman \& Schulman, 1986)

d) Soledad (Anderson, Horowitz, \& French, 1983)

e) Mal estado de salud física (Peterson, Seligman \& Vaillant, 1988)

De esta manera las causas que según los ingresantes actúan como impulsores o posibles causas para su ingreso a la universidad serán estudiados en las tres dimensiones del estilo explicativo: internalidad, estabilidad y globalidad, para posteriormente categorizarlas en estilo atribucional optimista o pesimista.

En la presente investigación hemos trabajado las siguientes hipótesis:

H1 Los estudiantes que expresan su deseo de cambiarse de Escuela Académico Profesional difieren en sus expectativas hacia la profesión elegida respecto a los estudiantes que expresan su deseo de mantenerse hasta concluir sus estudios en la especialidad a la que ingresaron. 
H2 Los estudiantes que expresan su deseo de cambiarse de Escuela Académico Profesional difieren en sus percepciones hacia la profesión elegida respecto a los estudiantes que expresan su deseo de mantenerse hasta concluir sus estudios en la especialidad a la que ingresaron.

H3 Los estudiantes que expresan su deseo de cambiarse de Escuela Académico Profesional difieren en sus estilos atribucionales de las causas que determinaron su ingreso a la universidad respecto a los estudiantes que expresan su deseo de mantenerse hasta concluir sus estudios en la especialidad a la que ingresaron.

H4 Según el género difieren las expectativas, percepciones y estilos atribucionales en los estudiantes que expresan su voluntad de traslado a otra especialidad respecto a los que. manifiestan su voluntad de permanecer en la Escuela Académico Profesional a la que ingresaron.

H5 Según la Escuela Académico Profesional a la que se ingresó difieren las expectativas, percepciones y estilos atribucionales en los estudiantes que expresan su voluntad de traslado de sus pares que manifiestan permanecer en la especialidad a la que ingresaron.

Las variables con las que trabajamos en el presente estudio son:

Variable Independiente: Deseo de traslado o de permanencia en la Escuela Académico Profesional, el sexo y la Escuela Académico Profesional a la que se ingresó.

Variable Dependiente: Expectativas, percepciones y estilo atribucional.

Variable control: Ingresantes a la UNMSM. En el concurso de admisión 2000.

Queremos por lo señalado alcanzar los siguientes objetivos:

- Explorar e identificar la concordancia entre la Facultad a la que ingresó el estudiante con las expectativas y percepciones vocacionales.

- Identificar la concordancia entre la Facultad a la que ingresó el estudiante con los estilos atribucionales que determinaron el ingreso a la Universidad.

- Identificar el papel del género y la Facultad académica de pertenencia sobre las expectativas y percepciones vocacionales y estilos atribucionales sobre la permanencia o deserción académica.

\section{METODO}

La presente investigación es descriptiva comparativa en cuanto se pretende conocer las expectativas, percepciones y estilos atribucionales entre los que ingresando a la Universidad a una determinada Escuela Académico Profesional, desean trasladarse a otra especialidad, respecto a sus pares que expresan su conformidad y firme propósito de permanecer en ella.

La recolección de información fue colectiva en grupos no mayores de 25 por vez.

La técnica que se utilizó fue fundamentalmente la psicométrica, con excepción de pocos casos donde fue necesaria la entrevista a alumnos que en las pruebas aplicadas se detectó alto índice de inconsistencias que actúa negativamente sobre la validez y confiabilidad de los resultados.

Para el examen psicométrico se utilizó dos pruebas tipo cuestionario, el primero es un cuestionario de expectativas y percepciones vocacionales con relación a la escuela académico profesional al que se ingresó, el cuestionario permite identificar el perfil del 
estudiante en relación a sus expectativas, información previa y grado de identificación con la profesión que pretende, en ese sentido se podrá detectar el grado de coherencia entre la plaza estudiantil alcanzada y sus expectativas. La validez del cuestionario fue por el método de contenido por el criterio de Jueces, reteniendo solo aquellos ítemes que resultaron significativos al 0.05 de riesgo de error.

La confiabilidad del cuestionario fue por el método del test-retest aplicado a una muestra de 150 estudiantes de diferentes programas académicos, ingresantes 2000 elegidos aleatoriamente el coeficiente de correlación entre la primera y la segunda aplicación la misma que se efectúo con un intervalo de un mes, fue de 0,81 y un índice de fiabilidad del 0.90 .

La segunda prueba para medir los estilos atribucionales de las causas que determinaron el Ingreso a la Universidad se utilizó el Cuestionario de Estilo Atribucional (ASQ: Atributional Style Quesstionnaire), adaptado a las condiciones de Ingreso a la Universidad manteniendo constante los itemes que evalúan: Internalidad, direccionalidad y globalidad, cuya validez de constructo ha sido demostrada por sus autores Peterson, y Villanova 1988 y la confiabilidad revisada para el presente estudio fue por el método de consistencia interna encontrado coeficientes que van desde un mínimo de 0.89 hasta 0.95.

\section{POBLACIÓN Y MUESTRA.}

La muestra fue al 0.05 de margen de error y un nivel de confianza del 95\% estimada a partir de 6000 ingresantes a la UNMSM, en el año lectivo 2000, distribuidos en 19 Facultades y éstas a su vez en 48 E.A.P., estamos hablando de un registro de aproximadamente 6000 examinados. La muestra está constituida por 698 estudiantes aleatoriamente seleccionados de 20 Escuelas Académico Profesional. Es conveniente señalar que algunas escuelas aleatoriamente seleccionadas no fue posible examinarlas debido a la nula colaboración de las autoridades de turno, situación que nos llevó al reemplazamiento aleatorio.

\section{Los procedimientos seguidos fueron:}

En primer lugar se prepararon los materiales para la aplicación piloto que concluyó con la verificación de la confiabilidad y validez de los instrumentos utilizados.

En segundo lugar se depuraron los protocolos con el propósito de eliminar aquellos que resultaron inconsistentes o intencionalmente mal contestados.

En tercer lugar se elaboró la matriz consolidado para la configuración de la base de datos.

El análisis estadístico fue descriptivo e inferencial comparativo utilizando la prueba Ji cuadrado, la razón crítica de la diferencia de porcentajes y la contrastación de promedios.

Adicionalmente se calcularon estadísticos descriptivos para caracterizar a la muestra estudiada en función a sus expectativas y percepciones sobre su vocación, lo propio se hizo con los estilos atribucionales de las causas que determinaron el ingreso a la Universidad.

\section{RESULTADOS}

El análisis de resultados parte de la identificación de estudiantes que expresan su voluntad de trasladarse a una Escuela Académico Profesional distinta a la que ingresaron respecto a los estudiantes que expresan su deseo de mantenerse hasta culminar sus estudios.

Conformados los grupos distribuidos por cada escuela académico profesional y 
género se calculó la asociación con las expectativas y las percepciones y se calculó las diferencias de medias aritméticas para el estilo atribucional, complementariamente se elaboraron tablas de ordenación con el propósito de describir la posición que ocupan las razones de cambio, expectativas y percepciones que a continuación detallamos.

1. Expectativas de los estudiantes que expresan su voluntad de permanecer respecto a quienes comunican su deseo de trasladarse de escuela académico profesional, para abordar este punto primero clasificamos a la muestra examinada en función al deseo expresado de trasladarse o no a otra E.A.P., que a continuación presentamos en la siguiente tabla:

\section{Tabla 1}

De frecuencia absolutas de alumnos que expresan su deseo de trasladarse a otra escuela académico profesional (SI) respecto a quienes expresan su deseo de concluir sus estudios en la escuela académico profesional a la que ingresaron (NO).

\begin{tabular}{|l|c|c|c|c|}
\hline \multirow{2}{*}{ Escuela } & \multicolumn{2}{|c|}{ Vescripción } & \multicolumn{2}{c|}{ Mujer } \\
\cline { 2 - 5 } & $\mathbf{S i}$ & $\mathbf{N o}$ & $\mathbf{S i}$ & $\mathbf{N o}$ \\
\hline Contabilidad & 8 & 28 & 7 & 49 \\
Administración & 3 & 12 & 2 & 14 \\
Neg. Internacional & 3 & 18 & 5 & 14 \\
M Veterinaria & 9 & 49 & 6 & 48 \\
Educación & 5 & 11 & 13 & 15 \\
Economía & 6 & 9 & 3 & 6 \\
Ing. Eléctrica & 4 & 14 & 3 & 1 \\
Ing. Electrónica & 2 & 11 & 0 & 4 \\
Inv. Operativa & 5 & 10 & 7 & 7 \\
Estadistíca & 5 & 2 & 1 & 4 \\
Matematíca & 3 & 7 & 5 & 12 \\
CC. Biológicas & 2 & 7 & 3 & 12 \\
Com. Social & 3 & 8 & 1 & 2 \\
Arte & 4 & 2 & 0 & 5 \\
Bibliotecología & 0 & 1 & 0 & 0 \\
Literatura & 0 & 1 & 0 & 1 \\
Psicología & 7 & 20 & 10 & 54 \\
Linguistíca & 1 & 1 & 0 & 2 \\
Odontología & 5 & 6 & 3 & 9 \\
Far. y Bioquímica & 6 & 19 & 6 & 47 \\
\hline Tot. Absolutos & $\mathbf{8 1}$ & $\mathbf{2 3 6}$ & $\mathbf{7 5}$ & $\mathbf{3 0 6}$ \\
Porcentajes & $\mathbf{1 1 . 6 0}$ & $\mathbf{3 3 . 8 1}$ & $\mathbf{1 0 . 7 4}$ & $\mathbf{4 3 . 8 4}$ \\
\hline
\end{tabular}

Del cuadro se desprende que el $22 \%$ de los examinados expresan su deseo de cambiarse a otra especialidad, frente al $78 \%$ que expresa su vivo deseo de permanecer hasta culminar exitosamente, al comparar los porcentajes la diferencia es significativa aún para el 0.01 de error a favor de la permanencia hallando un valor Ji cuadrado de 31.36. Sin embargo este 22 por ciento, indica que aproximadamente 1320 ingresantes a las diferentes E.A.P., tienen en mente solicitar su traslado a otra especialidad profesional.

Luego con estos grupos se elaboraron cuadros de contingencia para calcular la asociación entre la voluntad o no de traslado a otra Escuela Académico Profesional con las expectativas hacia la especialidad profesional a la que ingresaron y se consideró oportuno efectuar el análisis en función al género y para la muestra total, los resultados se reproducen a continuación: 
Tabla 2

De contingencia de frecuencias absolutas correspondiente a la muestra de varones en función al deseo de traslado de especialidad y expectativas profesionales

\begin{tabular}{|l|c|c|c|}
\cline { 2 - 4 } \multicolumn{1}{c|}{} & $\begin{array}{c}\text { Desean } \\
\text { Trasladarse }\end{array}$ & $\begin{array}{c}\text { No desean } \\
\text { Trasladarse }\end{array}$ & Total \\
\hline Pasiva & 20 & 25 & 45 \\
Activa & 20 & 40 & 60 \\
Armónica & 41 & 171 & 212 \\
\hline \multicolumn{1}{|c|}{ Total } & $\mathbf{8 1}$ & $\mathbf{2 3 6}$ & $\mathbf{3 1 7}$ \\
\hline
\end{tabular}

Con las frecuencias de la tabla el valor de Ji cuadrado para la muestra de varones es de 14.65 muy significativa aún para el 0,01 de margen de error con un valor en el coeficiente $V$ de Cramer de 0.21 lo que indica que la asociación hallada no es producto del azar. Se puede leer de la tabla que la dirección de la asociación está hacia la expectativa donde confluyen las metas y las acciones pertinentes con el deseo expresado de permanecer en ,la especialidad a la que se ingresó.

Tabla 3

De contingencia de frecuencias absolutas correspondiente a la muestra de mujeres en función al deseo de traslado de especialidad y expectativas profesionales

\begin{tabular}{|l|c|c|c|}
\cline { 2 - 4 } \multicolumn{1}{c|}{} & $\begin{array}{c}\text { Desean } \\
\text { Trasladarse }\end{array}$ & $\begin{array}{c}\text { No desean } \\
\text { Trasladarse }\end{array}$ & Total \\
\hline Pasiva & 28 & 20 & 48 \\
Activa & 30 & 50 & 80 \\
Armónica & 17 & 236 & 253 \\
\hline Total & $\mathbf{7 5}$ & $\mathbf{3 0 6}$ & $\mathbf{3 8 1}$ \\
\hline
\end{tabular}

Con las frecuencias de la tabla el valor de Ji cuadrado para la muestra de mujeres es de 26.91 muy significativa aún para el 0,01 de margen de error con un valor en el coeficiente $V$ de Cramer de 0.26 lo que indica que la asociación hallada no es producto del azar. Se puede leer de la tabla, que la dirección de la asociación, está hacia la expectativa donde confluyen las metas y las acciones pertinentes, con el deseo expresado de permanecer en la especialidad a la que se ingresó.

\section{Tabla 4}

De contingencia de frecuencias absolutas correspondiente a la muestra TOTAL en función al deseo de traslado de especialidad y expectativas profesionales

\begin{tabular}{|l|c|c|c|}
\cline { 2 - 4 } \multicolumn{1}{c|}{} & $\begin{array}{c}\text { Desean } \\
\text { Trasladarse }\end{array}$ & $\begin{array}{c}\text { No desean } \\
\text { Trasladarse }\end{array}$ & Total \\
\hline Pasiva & 48 & 45 & 93 \\
Activa & 50 & 90 & 140 \\
Armónica & 58 & 407 & 465 \\
\hline Total & $\mathbf{1 5 6}$ & $\mathbf{5 4 2}$ & $\mathbf{6 9 8}$ \\
\hline
\end{tabular}

Con las frecuencias de la tabla el valor de Ji cuadrado para la muestra total es de 26.14 muy significativa aún para el 0,01 de margen de error con un valor en el coeficiente V de Cramer de 0.20 lo que in-dica que la asociación hallada no es producto del azar. Se puede leer de la tabla que la dirección de la asociación está hacia la expectativa donde 
confluyen las metas con las acciones pertinentes con el deseo expresado de permanecer en la especialidad a la que se ingresó representado al $58 \%$.

De los resultados se puede afirmar que los estudiantes que expresan su deseo de mantenerse hasta concluir sus estudios en la especialidad a la que ingresaron difieren en sus expectativas de sus pares que expresan su deseo de cambiarse de Escuela Académico Profesional.

2. Percepciones de los estudiantes que expresan su voluntad de permanecer respecto a quienes comunican su deseo de trasladarse de Escuela Académico Profesional, para efectuar este análisis hemos repetido la secuencia anterior, con la diferencia que las percepciones solo presentan dos categorías, la primera de significados favorables y la segunda de significados desfavorables hacia la especialidad a la que ingresaron, los resultados pueden verse en las siguientes tablas de contingencia:

\section{Tabla 5}

De contingencia de frecuencias absolutas correspondiente a la muestra de varones en función al deseo de traslado de especialidad y percepciones profesionales

\begin{tabular}{|l|c|c|c|}
\cline { 2 - 4 } \multicolumn{1}{c|}{} & $\begin{array}{c}\text { Desean } \\
\text { Trasladarse }\end{array}$ & $\begin{array}{c}\text { No desean } \\
\text { Trasladarse }\end{array}$ & Total \\
\hline Desfavora. & 40 & 66 & 106 \\
Favorable & 41 & 170 & 211 \\
\hline Total & $\mathbf{8 1}$ & $\mathbf{2 3 6}$ & $\mathbf{3 1 7}$ \\
\hline
\end{tabular}

Con las frecuencias de la tabla el valor de Ji cuadrado para la muestra varones es de 12.43 muy significativa aún para el 0,0 1 de margen de error con un valor en el coeficiente $V$ de Cramer de 0.20 lo que indica que la asociación hallada no es producto del azar. Se puede leer de la tabla que la dirección de la asociación está hacia la percepción de significado favorable con la especialidad elegida en dirección a los estudiantes que expresan el deseo de permanecer en la especialidad a la que ingresaron representando al $54 \%$.

Tabla 6

De contingencia de frecuencias absolutas correspondiente a la muestra de mujeres en función al deseo de traslado de especialidad y percepciones profesionales

\begin{tabular}{|l|c|c|c|}
\cline { 2 - 4 } \multicolumn{1}{c|}{} & $\begin{array}{c}\text { Desean } \\
\text { Trasladarse }\end{array}$ & $\begin{array}{c}\text { No desean } \\
\text { Trasladarse }\end{array}$ & Total \\
\hline Desfavora. & 38 & 89 & 127 \\
Favorable & 37 & 217 & 254 \\
\hline Total & $\mathbf{7 5}$ & $\mathbf{3 0 6}$ & $\mathbf{3 8 1}$ \\
\hline
\end{tabular}

Con las frecuencias de la tabla el valor de Ji cuadrado para la muestra mujeres es de 12.62 muy significativa aún para el 0,01 de margen de error con un valor en el coeficiente $V$ de Cramer de 0.19 lo que indica que la asociación hallada no es producto del azar. Se puede leer de la tabla que la dirección de la asociación está hacia la percepción de significado favorable con la especialidad elegida en dirección a los estudiantes que expresan el deseo de permanecer en la especialidad a la que ingresaron representando al $57 \%$. 
Tabla 7

De contingencia de frecuencias absolutas correspondiente a la muestra TOTAL en función al deseo de traslado de especialidad y percepciones profesionales

\begin{tabular}{|l|c|c|c|}
\cline { 2 - 4 } \multicolumn{1}{c|}{} & $\begin{array}{c}\text { Desean } \\
\text { Trasladarse }\end{array}$ & $\begin{array}{c}\text { No desean } \\
\text { Trasladarse }\end{array}$ & Total \\
\hline Desfavora. & 78 & 155 & 233 \\
Favorable & 78 & 387 & 465 \\
\hline Total & $\mathbf{1 5 6}$ & $\mathbf{5 4 2}$ & $\mathbf{6 9 8}$ \\
\hline
\end{tabular}

Con las frecuencias de la tabla el valor de Ji cuadrado para la muestra total es de 24 . 95 muy significativa aún para el 0,01 de margen de error con un valor en el coeficiente $\mathrm{V}$ de Cramer de 0.20 lo que indica que la asociación hallada no es producto del azar. Se puede leer de la tabla que la dirección de la asociación está hacia la percepción de significado favorable con la especialidad elegida en dirección a los estudiantes que expresan el deseo de permanecer en la especialidad a la que ingresaron representando al $55 \%$.

De lo hallado se desprende que efectivamente los estudiantes que expresan su deseo de mantenerse hasta concluir sus estudios en la especialidad a la que ingresaron difieren en sus percepciones hacia la profesión elegida respecto a los estudiantes que expresan su deseo de cambiarse de escuela académico profesional, siendo la percepción favorable hacia la especialidad de ingreso, en los estudiantes que no presentan intención de traslado.

2. Estilos atribucionales de los estudiantes que expresan su voluntad de permanecer respecto a quienes comunican su deseo de trasladarse de Escuela Académico Profesional, para efectuar este análisis hemos contrastado mediante la razón crítica de diferencia de medias, correspondientes a los estudiantes con deseo y sin deseo expresado de traslado, en cada una de las dimensiones del estilo atribucional: Internoexterno, Inestable--estable y específico-global, que a continuación desarrollamos.

3. 3.1 La dimensión Interno-Externo, del estilo atribucional, al efectuar las comparaciones en el grupo de varones y de mujeres en relación al deseo (SI) o no (NO) de traslado a otra especialidad, se observa que no hay diferencias significativas, lo que indica que esta dimensión del estilo atribucional no es típico en ninguno de los grupos examinados. Sin embargo es conveniente indicar que los varones y las mujeres que no expresan deseo de traslado son similares en esta dimensión con una ligera tendencia hacia la internalidad. Por su parte las mujeres que expresan deseo de traslado presentan un promedio de internalidad de 56.65, frente al 51. 27 de los varones, cuya diferencia de 5.38 arroja un valor t de 2.91 muy significativa aún para el 0.01 de margen de error, lo que significa que las mujeres con deseo de traslado a otra especialidad se caracterizan por agruparse hacia la internalidad, los datos pueden verse a continuación. 
Tabla 8

De Diferencia de promedios del factor internalidad-externalidad del estilo atribucional, en función al género y por subgrupos según el deseo expresado de NO y SI traslado de especialidad profesional

\begin{tabular}{|l|c|c|c|c|}
\cline { 2 - 5 } \multicolumn{1}{c|}{} & \multicolumn{2}{c|}{ Hombres } & \multicolumn{2}{c|}{ Mujeres } \\
\cline { 2 - 5 } \multicolumn{1}{c|}{} & NO & SI & NO & SI \\
\hline Número & 235 & 80 & 305 & 74 \\
Media & 54.3 & 51.27 & 54.67 & 56.65 \\
Mediana & 55 & 50 & 55 & 51 \\
Asimetría & -0.32 & -.048 & -0.02 & -0.63 \\
Varianza & 113.12 & 154.7 & 90.86 & 109.8 \\
\hline Dif. medias & \multicolumn{2}{|c|}{3,55} & \multicolumn{2}{|c|}{1,97} \\
Error estánd & \multicolumn{2}{|c|}{1,94} & \multicolumn{2}{|c|}{1,33} \\
Puntaje t. & \multicolumn{2}{|c|}{1,96} & \multicolumn{2}{c|}{1,48} \\
tal 0.05 & \multicolumn{2}{|c|}{1,96} & \multicolumn{2}{c|}{1,96} \\
Decisión & \multicolumn{2}{|c|}{ Ho } & \multicolumn{2}{c|}{ Ho } \\
\hline
\end{tabular}

3.2 La dimensión Inestabilidad-Estabilidad, del estilo atribucional, al efectuar las comparaciones en el grupo de varones y de mujeres con relación al deseo (SI) o no (NO) de traslado a otra especialidad, se observa que no hay diferencias significativas, lo que indica que esta. dimensión del estilo atribucional no es típico en ninguno de los grupos examinados orientándose hacia la tendencia hacia la inestabilidad con excepción de las mujeres que expresan deseo de traslado quienes se orientan hacia la tendencia a la estabilidad, los datos pueden verse a continuación.

Tabla 9

De Diferencia de promedios del factor Inestabilidad-estabilidad del estilo atribucional, en función al género y por subgrupos según el deseo expresado de NO y SI traslado de especialidad profesional

\begin{tabular}{|c|c|c|c|c|}
\cline { 2 - 5 } \multicolumn{1}{c|}{} & \multicolumn{2}{c|}{ Hombres } & \multicolumn{2}{c|}{ Mujeres } \\
\cline { 2 - 5 } \multicolumn{1}{c|}{} & NO & SI & NO & SI \\
\hline Número & 255 & 107 & 281 & 119 \\
Media & 44.94 & 44.38 & 45.20 & 47.02 \\
Mediana & 45 & 44 & 46 & 47 \\
Asimetría & -0.00 & -0.05 & -0.01 & 0.16 \\
Varianza & 158.63 & 115.86 & 139.62 & 101.22 \\
\hline Dif. media & \multicolumn{2}{|c|}{0,56} & \multicolumn{2}{c|}{1,82} \\
Error estánd & \multicolumn{2}{|c|}{1,31} & \multicolumn{2}{|c|}{1,16} \\
Puntaje t. & \multicolumn{2}{c|}{0,43} & \multicolumn{2}{c|}{1,57} \\
ta10.05 & \multicolumn{2}{c|}{1,96} & \multicolumn{2}{c|}{1,96} \\
Decisión & \multicolumn{2}{c|}{ Ho } & \multicolumn{2}{c}{ Ho } \\
\hline
\end{tabular}


3.3 La dimensión Especificidad-Globalidad, del estilo atribucional, al efectuar las comparaciones en el grupo de varones y de mujeres con relación al deseo (SI) o no (NO) de traslado a otra especialidad, se observa que no hay diferencias significativas, lo que indica que esta dimensión del estilo atribucional no es típico en ninguno de los grupos examinados. En esta dimensión a diferencia de las dos anteriores el promedio de los examinados en general se ubica dentro de la especificidad, los datos pueden verse a continuación.

Tabla 10

De Diferencia de promedios del factor Especificidad-Globalidad del estilo atribucional, en función al género y por subgrupos según el deseo expresado de NO y SI traslado de especialidad profesional

\begin{tabular}{|c|c|c|c|c|}
\hline & \multicolumn{2}{|c|}{ Hombres } & \multicolumn{2}{|c|}{ Mujeres } \\
\hline & $\mathrm{NO}$ & $S I$ & NO & SI \\
\hline Número & 234 & 81 & 305 & 74 \\
\hline Media & 42.53 & 41.94 & 41.65 & 42.39 \\
\hline Mediana & 43 & 42 & 42 & 41.5 \\
\hline Asimetría & 0.13 & 0.005 & -0.03 & 0.67 \\
\hline Varianza & 11328 & 128.16 & 121.31 & 112.57 \\
\hline Dif. medias & \multicolumn{2}{|c|}{0,56} & \multicolumn{2}{|c|}{0,74} \\
\hline Error estánd & \multicolumn{2}{|c|}{1,47} & \multicolumn{2}{|c|}{1,38} \\
\hline Puntaje t. & \multicolumn{2}{|c|}{0,40} & \multicolumn{2}{|c|}{0,54} \\
\hline tal 0.05 & \multicolumn{2}{|c|}{1,96} & \multicolumn{2}{|c|}{1,96} \\
\hline Decisión & \multicolumn{2}{|c|}{ Ho } & \multicolumn{2}{|c|}{ Ho } \\
\hline
\end{tabular}

De lo hallado se desprende que, los estudiantes que expresan su deseo de cambiarse de Escuela Académico Profesional no difieren en sus estilos atribucionales de las causas que determinaron su ingreso a la universidad respecto a los estudiantes que expresan su deseo de mantenerse hasta concluir sus estudios en la especialidad a la que ingresaron. Sin embargo es conveniente señalar que sólo en la dimensión internalidad-externalidad las mujeres que desean trasladarse de especialidad presentan mayor intemalidad.

El estilo atribucional optimista se caracteriza cuando las personas obtienen la siguiente combinación: Externalidad Inestabilidad y Especificidad, estos sujetos asumen que las causas que en alguna medida explican lo que les sucede son debido a otras causas o situaciones que debido a sí mismos, que las causas negativas no estarán presentes nunca más, y que su acción solo influye en una determinada situación.

El estilo atribucional pesimista se caracteriza cuando las personas obtienen la siguiente combinación: Internalidad Estabilidad y Globalidad, estos sujetos asumen que las causas que en alguna medida explican lo que les sucede son totalmente debido a sí mismos, que las causas negativas siempre estarán presentes, y que su acción influye en todas las situaciones de su vida.

En la muestra examinada, encontramos que tanto los varones como las mujeres que expresan permanecer en la Escuela Académico Profesional a la que ingresaron tienden a la internalidad, dejando entrever alguna posibilidad para la frustración y desmoralización, que es más acentuado en las mujeres que desean trasladarse. Sin embargo el panorama no es del todo malo ya que todos los examinados tienden a la inestabilidad, es decir asumen que las 
causas de acontecimientos negativos solo ocurren una vez y nunca más estarán presentes agregando que en todo caso las causas de lo malo solo actúan sobre una situación concreta sin influir a todas las situaciones de la vida. Hecho que localiza a la dimensión internalidadexternalidad del estilo atribucional como la que tendrá que trabajarse, para definir a nuestros jóvenes con estilo atribucional optimista, el estudio revela que hay solo tendencia al encontrar dos dimensiones favorables.

4. El género y las expectativas, percepciones y estilos atribucionales.- En esta sección nos proponemos averiguar el papel que juega el sexo de los ingresantes que expresan su deseo de traslado, o su deseo de permanecer en la especialidad a la que ingresaron; para lo cual se analizaran los datos primero en las expectativas, luego en las percepciones para concluir con el estilo atribucional.

4.1. Las expectativas hacia la especialidad profesional a la que se ingreso en función al sexo, para este propósito hemos tabulado para cada categoría de expectativa a los varones y mujeres que expresan su voluntad de traslado y con la prueba del Ji cuadrado vemos si la predominancia de posibles trasladantes es significativa en función al sexo. Realizado el análisis encontramos que las expectativas pasivas de los varones no es significativamente mayor que el grupo de la mujeres. Lo mismo encontramos con las expectativas activas. Por su parte al analizar las expectativas armónicas, el número de varones con deseo de traslado es mayor significativamente que el grupo de mujeres, lo que deja entrever que bajo condiciones de traslado los varones tienden a compatibilizar mejor sus ideas o metas con las conductas pertinentes al logro. En la parte inferior de la tabla que a continuación se reproduce hemos calculado el Ji cuadrado para la frecuencia de varones que desean traslado frente al grupo de mujeres encontrando que el Ji calculado es espúreo al igual que la intensidad de la asociación calculada con la $V$ de Cramer, lo que nos dice que la demanda de traslado de especialidad no es exclusividad de uno de los sexos a lo que agregamos que el porcentaje de varones con expectativas armónicas y que expresan deseo de traslado es del $51 \%$ sin marcar notoria discrepancia con sus similares que se encuentran en la expectativa pasiva y activa, por ende la diferencia exogrupo hallada no es lo suficiente para afirmar que el género plantea diferencias en las expectativas hacia la especialidad profesional a la que ingresaron, tal como puede verse en el siguiente cuadro.

Tabla 11

De valores Ji cuadrado entre las categorías de expectativas con la voluntad de traslado de especialidad por el sexo de los estudiantes

\begin{tabular}{|l|c|c|c|c|}
\cline { 2 - 5 } \multicolumn{1}{c|}{} & Var. & Muj. & $\mathbf{X}^{2}$ & $\begin{array}{c}\text { V de } \\
\text { Cramer }\end{array}$ \\
\hline Pasiva & 20 & 28 & 1.33 & 0.16 \\
Activa & 20 & 30 & 2 & 0.20 \\
Armónica & 41 & 17 & 9.93 & 0.41 \\
GrupoTotal & 81 & 75 & 0.23 & 0.04 \\
\hline
\end{tabular}

4.2 Las Percepciones hacia la especialidad profesional a la que se ingresó en función al sexo, para este propósito de manera análoga al estudio anterior hemos tabulado para cada categoría de percepción a los varones y mujeres que expresan su voluntad de traslado y con la prueba del Ji cuadrado vemos si la predominancia de posibles trasladantes es significativa en función al sexo. Realizado el análisis encontramos que las percepciones 
desfavorables y las favorables de los varones no son significativamente mayores que en el grupo de las mujeres. Esto indica que las ideas o los significados que los ingresantes tienen de la profesión a la que ingresaron no es una causal del traslado, los resultados se presentan a continuación.

Tabla 12

De valores Ji cuadrado entre las categorías de percepciones con la voluntad de traslado de especialidad por el sexo de los estudiantes

\begin{tabular}{|l|c|c|c|c|}
\cline { 2 - 5 } \multicolumn{1}{c|}{} & Var. & Muj. & $\mathbf{X}^{2}$ & $\begin{array}{c}\text { V de } \\
\text { Cramer }\end{array}$ \\
\hline Desafavor. & 40 & 38 & 0.05 & 0.02 \\
Favorable & 41 & 37 & 0.21 & 0.05 \\
\hline
\end{tabular}

4.3. Los Estilos atribucionales de las causas de ingreso a la universidad hacia la especialidad profesional a la que se ingresó en función del sexo, para este propósito contrastamos las medias aritméticas de las dimensiones del estilo atribucional tabulado para cada dimensión en el grupo de varones y mujeres que expresan su voluntad o no de traslado y con la prueba de la razón crítica de diferencia de medias se llegó a los siguientes resultados

En la dimensión internalidad externalidad los varones que expresan su deseo de traslado de especialidad presentan menor internalidad que las mujeres. Por lo contrario quienes expresan su voluntad de permanecer no difieren entre sí caracterizándose por su tendencia a la internalidad tal como puede verse en la siguiente tabla.

Tabla 13

De Diferencia de promedios del factor Internalidad-externalidad del estilo atribucional, correspondiente a la muestra de estudiantes que expresan deseo y no de traslado de especialidad en función al género

\begin{tabular}{|l|c|c|c|c|}
\cline { 2 - 5 } \multicolumn{1}{c|}{} & \multicolumn{2}{c|}{ Expr. Tras. } & Expr. No Tras. \\
\cline { 2 - 5 } \multicolumn{1}{c|}{} & Varón & Mujer & Varón & Mujer \\
\hline Número & 80 & 74 & 235 & 306 \\
Media & 51.27 & 56.65 & 54.3 & 54.67 \\
Mediana & 50 & 57 & 55 & 55 \\
Asimetría & -0.48 & -0.63 & -0.32 & -0.02 \\
Varianza & 154.76 & 109.85 & 113.12 & 90.86 \\
\hline Dif. medias & \multicolumn{2}{|c|}{5,38} & \multicolumn{2}{c|}{0,37} \\
Error estánd & \multicolumn{2}{|c|}{1,85} & \multicolumn{2}{c|}{0,88} \\
Puntaje t. & \multicolumn{2}{c|}{2,91} & \multicolumn{2}{c|}{0,42} \\
tal 0.05 & \multicolumn{2}{c|}{1,96} & 1,96 \\
Decisión & \multicolumn{2}{|c|}{ Ho } & Ho \\
\hline
\end{tabular}

En la dimensión de Inestabilidad Estabilidad, los varones que expresan su deseo de traslado de especialidad no difieren de las mujeres. Por su parte los varones que expresan su voluntad de permanecer tampoco difieren de las mujeres con sentimiento 'similar ubicándose en la categoría de tendencia a la inestabilidad, tal como puede verse en la siguiente tabla. 
Tabla 14

De Diferencia de promedios del factor Inestabilidad-estabilidad del estilo atribucional, correspondiente a la muestra de estudiantes que expresan deseo y no de traslado de especialidad en función al género

\begin{tabular}{|l|c|c|c|c|}
\cline { 2 - 5 } \multicolumn{1}{c|}{} & \multicolumn{3}{c|}{ Expr. Tras. } & \multicolumn{2}{c|}{ Expr. No Tras. } \\
\cline { 2 - 5 } \multicolumn{1}{c|}{} & Varón & Mujer & Varón & Mujer \\
\hline Número & 107 & 119 & 255 & 281 \\
Media & 44.38 & 47.02 & 44.94 & 45.20 \\
Mediana & 44 & 47 & 45 & 46 \\
Asimetría & -0.05 & 0.16 & -0.00 & -0.01 \\
Varianza & 115.86 & 101.22 & 158.63 & 139.62 \\
\hline Dif. medias & \multicolumn{2}{|c|}{2,64} & \multicolumn{2}{|c|}{0,26} \\
Error estánd & \multicolumn{2}{|c|}{1,39} & \multicolumn{2}{c|}{1,06} \\
Puntaje t. & \multicolumn{2}{|c|}{1,89} & \multicolumn{2}{c|}{0,24} \\
tal 0.05 & \multicolumn{2}{|c|}{1,96} & \multicolumn{2}{c|}{1,96} \\
Decisión & \multicolumn{2}{|c|}{ Ho } & \multicolumn{2}{c|}{ Ho } \\
\hline
\end{tabular}

En la dimensión de Especificidad Globalidad del estilo atribucional, los varones que expresan su deseo de traslado de especialidad no difieren de las mujeres. Por su parte los varones que expresan su voluntad de permanecer tampoco difieren de las mujeres con sentimiento similar ubicándose en la categoría de especificidad, tal como puede verse en la siguiente tabla.

Tabla 15

De Diferencia de promedios del factor Especificidad-Globalidad del estilo atribucional, correspondiente a la muestra de estudiantes que expresan deseo y no de traslado de especialidad en función al género

\begin{tabular}{|l|c|c|c|l|}
\cline { 2 - 5 } \multicolumn{1}{c|}{} & \multicolumn{2}{c|}{ Expr. Tras. } & \multicolumn{2}{c|}{ Expr. No Tras. } \\
\cline { 2 - 5 } \multicolumn{1}{c|}{} & Varón & Mujer & Varón & Mujer \\
\hline Número & 81 & 74 & 234 & 305 \\
Media & 41.94 & 42.39 & 42.53 & 41.65 \\
Mediana & 42 & 41.5 & 43 & 42 \\
Asimetría & 0.005 & 0.67 & 0.13 & -0.03 \\
Varianza & 128.16 & 112.57 & 138.28 & 121.31 \\
\hline Dif. medias & \multicolumn{2}{|c|}{0,45} & \multicolumn{2}{c|}{0,88} \\
Error estánd. & \multicolumn{2}{|c|}{1,76} & \multicolumn{2}{c|}{0,99} \\
Puntaje t. & \multicolumn{2}{|c|}{0,26} & \multicolumn{2}{c|}{0,89} \\
tal 0.05 & \multicolumn{2}{c|}{1,96} & \multicolumn{2}{c|}{1,96} \\
Decisión & \multicolumn{2}{c|}{ Ho } & \multicolumn{2}{c}{ Ho } \\
\hline
\end{tabular}

Por los resultados tanto quienes expresan su deseo de traslado como los que expresan su deseo de permanecer en la especialidad profesional a la que ingresaron tienden al estilo de causalidad atribucional optimista, siendo la dimensión que requiere de trabajo urgente la internalidad, que puede llevar hacia la baja autoestima, la frustración y desmoralización, en síntesis el estilo atribucional no difiere en función al sexo. 
5. Las expectativas, percepciones y estilos atribucionales en función a la Escuela Académico Profesional de ingreso, para este punto hemos tabulado para el total de examinados por Escuela Académico Profesional, agrupados por su expreso deseo o no de traslado a otra especialidad profesional sus respuestas que lo ubican en expectativa armónica y percepción favorable, luego las analizamos con la prueba Ji cuadrado para establecer la asociación o no entre la expectativa, la percepción con la voluntad de traslado o permanencia, encontrando solo en once de las veinte E.A.P. asociaciones significativas de las expectativas y percepciones en los estudiantes que permanecerán respecto a la escuela académico profesional, en tanto que en nueve no hay asociaciones significativas. Al calcular el Ji cuadrado entre el número de escuelas académicos en las que hay asociación significativa con el número de escuelas académico profesionales, en las que no hay asociación, encontramos un valor calculado de 0.20 con un valor $\mathrm{V}$ de Cramer de 0.10 lo que indica que no hay asociación significativa entre la Escuela Académico a la que se ingresó con sus expectativas y percepciones en función a su deseo de traslado o permanencia tal como puede verse a continuación.

\section{Tabla 16}

\section{De Valores Ji cuadrado de las expectativas armónicas y percepciones favorables hacia la especialidad profesional de ingreso en función a la Escuela Académico Profesional en estudiantes que expresan su deseo de permanecer o trasladarse a otra especialidad profesional}

\begin{tabular}{|l|c|c|c|c|c|}
\hline Escuela Academ Profesion. & No.Part. & $\begin{array}{c}\text { Traslado } \\
\text { SI }\end{array}$ & $\begin{array}{c}\text { Traslado } \\
\text { NO }\end{array}$ & X2 & $\begin{array}{c}\text { Coefic. } \\
\text { V.Cramer }\end{array}$ \\
\hline Contabilidad & 92 & 15 & 77 & $41.78^{*}$ & 0.67 \\
Administración & 31 & 5 & 26 & $14.22^{*}$ & 0.68 \\
Negocios Internacionales & 40 & 8 & 32 & $14.4^{*}$ & 0.60 \\
Medicina veterinaria & 112 & 15 & 97 & $60.03^{*}$ & 0.73 \\
Educación & 44 & 18 & 26 & 1.78 & 0.20 \\
Economía & 24 & 9 & 15 & 1.5 & 0.25 \\
Eléctrica & 22 & 7 & 15 & 2.91 & 0.36 \\
Electrónico & 17 & 2 & 15 & $9.94^{*}$ & 0.76 \\
Investigación operativa & 29 & 12 & 17 & 0.86 & 0.17 \\
Estadística & 12 & 6 & 6 & 0.00 & 0.00 \\
Matemática & 27 & 8 & 19 & $4.48^{*}$ & 0.41 \\
Ciencias Biológicas & 24 & 5 & 19 & $8.17^{*}$ & 0.58 \\
Comunicación Social & 14 & 4 & 10 & 2.57 & 0.43 \\
Arte & 11 & 4 & 7 & 0.82 & 0.27 \\
Bibliotecología y Cc. Inform. & 41 & 0 & 1 & $10^{*}$ & 1 \\
Literatura & 20 & 0 & 2 & $20^{*}$ & 1 \\
Psicología & 91 & 17 & 74 & $35.7^{*}$ & 0.63 \\
Lingüística & 4 & 1 & 3 & 1 & 0.50 \\
Odontología & 23 & 8 & 15 & 2.13 & 0.30 \\
Farmacia y Bioquímica & 78 & 2 & 66 & $37.38^{*}$ & 0.69 \\
\hline
\end{tabular}

Por otro lado los estilos atribucionales fueron analizados tabulando el número de sujetos con estilo optimista de cada escuela académico profesional teniendo en cuenta su expresión de permanecer o trasladarse a otra especialidad profesional, no se encontró en ningún caso un valor Ji cuadrado significativo, por lo que se afirma que el estilo atribucional optimista o pesimista no es característico de una determinada Escuela Académico Profesional ni tampoco de los estudiantes que expresan su deseo de o no trasladarse a otra especialidad profesional. Estos resultados pueden verse en la siguiente tabla. 
Tabla 17

De Ji cuadrado del número de sujetos con estilo atribucional optimista pertenecientes a todas las Escuelas Académico Profesional estudiadas en función al deseo de o no trasladarse de especialidad profesional

\begin{tabular}{|c|c|c|c|c|c|}
\cline { 2 - 6 } \multicolumn{1}{c|}{} & No. Part. & $\begin{array}{c}\text { Traslado } \\
\text { SI }\end{array}$ & $\begin{array}{c}\text { Traslado } \\
\text { NO }\end{array}$ & X2 & $\begin{array}{c}\text { Coefic. } \\
\text { V Cramer }\end{array}$ \\
\hline $\begin{array}{l}\text { Estilo Optimista } \\
\text { todas las E.A.P. }\end{array}$ & 558 & 290 & 268 & 0.87 & 0.04 \\
$\begin{array}{c}\text { Estilo Pesimista } \\
\text { todas las E.A.P. }\end{array}$ & 140 & 78 & 62 & 1.83 & 0.11 \\
\hline
\end{tabular}

6. Complementariamente creemos conveniente presentar algunas tablas ordinales donde dando cuenta de las principales expectativas y percepciones hacia la profesión a la ingresaron.

Tabla 18

Ordenación de las razones por las que se quedarían estudiando esta profesión en función del género

\begin{tabular}{|c|c|c|}
\hline $\begin{array}{c}\text { Grados de } \\
\text { importancia }\end{array}$ & Varones & Mujeres \\
\hline ler. lugar & Vocación & Vocación \\
\hline 2 do-lugar & $\begin{array}{c}\text { Tiempo invertido } \\
\text { Futuro }\end{array}$ & $\begin{array}{c}\text { Puedo hacer } \\
\text { empresa }\end{array}$ \\
\hline 3er. lugar & Buena enseñanza & Razon de gusto \\
\hline 4to.lugar & $\begin{array}{c}\text { Elección adec. } \\
\text { Por solidaridad } \\
\text { Por rentabilidad } \\
\text { Ofrece oportunidades }\end{array}$ & Por prestigio \\
\hline
\end{tabular}

Tabla 19

De las razones por las que solicitarían traslado correspondiente ala muestra total y en función al género

\begin{tabular}{|l|l|l|}
\hline \multicolumn{1}{|c|}{$\begin{array}{c}\text { Grados de } \\
\text { importancia }\end{array}$} & \multicolumn{1}{|c|}{ Varones } & \multicolumn{1}{c|}{ Mujeres } \\
\hline 1er. lugar & $\begin{array}{l}\text { Vocación } \\
\text { No hay trabajo } \\
\text { Otras metas } \\
\text { Mala enseñanza }\end{array}$ & $\begin{array}{l}\text { Vocación } \\
\text { Por oportunid. }\end{array}$ \\
\hline 2do. lugar & Por oportunid. & Mala enseñanza \\
\hline 3er. lugar & Por prestigio & Por prestigio \\
\hline 4to. lugar & Rentabilidad & Otras metas \\
\hline
\end{tabular}

Los estudiantes que expresan su voluntad de trasladarse a otra Escuela Académico Profesional tienen como opciones preferenciales las siguientes:

Medicina humana, ingeniería de sistemas entre las dos primeras. (Ver tabla 20) 
Tabla 20

De las escuelas académico profesiona.les a las que les gustaría trasladarse correspondiente a la muestra total y en función al género

\begin{tabular}{|l|c|c|}
\hline \multicolumn{1}{|c|}{$\begin{array}{c}\text { Grados de } \\
\text { importancia }\end{array}$} & Varones & Mujeres \\
\hline ler. lugar & Med.Humana & Med.Humana \\
\hline 2do. lugar & Ing. Sistemas & Literatura \\
\hline 3er. lugar & $\begin{array}{c}\text { Publicidad } \\
\text { Tecn. Médica } \\
\text { Ter. Ocupacio. } \\
\text { Ed. Física } \\
\text { Arte } \\
\text { Com.Social } \\
\text { Electrónica }\end{array}$ & \\
\hline 4to. lugar & $\begin{array}{c}\text { Derecho } \\
\text { Rentabilidad }\end{array}$ & Turismo \\
\hline
\end{tabular}

Tabla 21

Razones jerarquizadas expresadas por las que las personas eligen y estudian una profesión correspondiente a la muestra total y en función al género.

\begin{tabular}{|l|c|c|}
\hline $\begin{array}{c}\text { Grados de } \\
\text { importancia }\end{array}$ & Varones & Mujeres \\
\hline ler. lugar & $\begin{array}{c}\text { Con esta profesión podré } \\
\text { realizar mis metas y } \\
\text { expectativas de vida }\end{array}$ & $\begin{array}{c}\text { Estudio esta profesión por el gran } \\
\text { esfuerzo que demanda. }\end{array}$ \\
\hline 2do. lugar & $\begin{array}{c}\text { Porque esta profesión me resulta } \\
\text { un reto por lo difícil que es. }\end{array}$ & $\begin{array}{c}\text { Porque esta profesión me resulta } \\
\text { un reto por lo difícil que es. }\end{array}$ \\
\hline 3er. lugar & $\begin{array}{c}\text { Estudio esta profesión por el } \\
\text { gran esfuerzo que demanda }\end{array}$ & $\begin{array}{c}\text { Estudio esta profesión por el gran } \\
\text { esfuerzo que demanda }\end{array}$ \\
\hline 4to. lugar & $\begin{array}{c}\text { Esta profesión me permitirá } \\
\text { ampliar mi red de amistades y } \\
\text { relaciones sociales }\end{array}$ & $\begin{array}{c}\text { Esta profesión me permitirá } \\
\text { ampliar mi red de amistades y } \\
\text { relaciones sociales. }\end{array}$ \\
\hline to. lugar & $\begin{array}{c}\text { Esta profesión me permitirá } \\
\text { tener el control hacia los demás }\end{array}$ & $\begin{array}{c}\text { Esta profesión me permitirá tener } \\
\text { el control hacia los demás }\end{array}$ \\
\hline
\end{tabular}


Tabla 22

Auto percepción de la habilidad o aptitud que lo caracteriza correspondiente muestra total y en función de género

\begin{tabular}{|c|c|c|}
\hline $\begin{array}{c}\text { Grado de } \\
\text { importancia }\end{array}$ & Varones & Mujeres \\
\hline 1er. lugar & $\begin{array}{c}\text { Resolver problemas } \\
\text { matemáticos }\end{array}$ & $\begin{array}{c}\text { Concentración a voluntad } \\
\text { para estudiar. }\end{array}$ \\
\hline 2do.lugar & $\begin{array}{c}\text { Concentración a voluntad } \\
\text { para estudiar }\end{array}$ & $\begin{array}{c}\text { Resolver problemas } \\
\text { matemáticos }\end{array}$ \\
\hline 3er. lugar & $\begin{array}{c}\text { Análisis de textos } \\
\text { científicos }\end{array}$ & Análisis de textos científicos \\
\hline 4to. lugar & $\begin{array}{c}\text { Resistencia a la fatiga o } \\
\text { cansancio para trabajar } \\
\text { en largas jornadas. }\end{array}$ & $\begin{array}{c}\text { Resistencia a la fatiga o } \\
\text { cansancio para trabajar en } \\
\text { largas jornadas. }\end{array}$ \\
\hline 5to. lugar & $\begin{array}{c}\text { Redactar o escribir } \\
\text { composiciones o ensayos }\end{array}$ & $\begin{array}{c}\text { Redactar o escribir } \\
\text { composiciones o ensayos }\end{array}$ \\
\hline
\end{tabular}

Tabla 23

Auto percepción de la autopercepción de personalidad que es lo caracteriza corrspondiente a la muestra total y en función del género

\begin{tabular}{|c|c|c|}
\hline $\begin{array}{c}\text { Grado de } \\
\text { importancia }\end{array}$ & Varones & Mujeres \\
\hline 1er. lugar & $\begin{array}{c}\text { El reconocimiento a mi esfuerzo } \\
\text { me estimula a continuar hacia } \\
\text { adelante }\end{array}$ & $\begin{array}{c}\text { El reconocimiento a mi } \\
\text { esfuerzo me estimulas a } \\
\text { continuar hacia adelante }\end{array}$ \\
\hline 2do.lugar & $\begin{array}{c}\text { Siempre escucho a las personas } \\
\text { opinando solo si es necesario }\end{array}$ & $\begin{array}{c}\text { Siempre escucho a las personas } \\
\text { opinando si es necesario }\end{array}$ \\
\hline 3er. lugar & $\begin{array}{c}\text { Los fracasos me impulsan a } \\
\text { encontrar otras soluciones }\end{array}$ & $\begin{array}{c}\text { Los fracasos me impulsan a } \\
\text { encontrar otras soluciones }\end{array}$ \\
\hline 4to.lugar & $\begin{array}{c}\text { Prefiero trabajar solo, la } \\
\text { presencia de otros me } \\
\text { desconcentra }\end{array}$ & $\begin{array}{c}\text { Prefiero trabajar solo, la } \\
\text { presencia de otros me } \\
\text { desconcentra }\end{array}$ \\
\hline 5to. lugar & $\begin{array}{c}\text { Me resulta fácil ocultar algunos } \\
\text { errores }\end{array}$ & $\begin{array}{c}\text { Me resulta fácil ocultar } \\
\text { algunos errores }\end{array}$ \\
\hline
\end{tabular}




\section{Tabla 24}

Auto de situaciones en las que s e siente cómodos, en función del género

\begin{tabular}{|l|l|l|}
\hline \multicolumn{1}{|c|}{$\begin{array}{c}\text { Grado de } \\
\text { importancia }\end{array}$} & \multicolumn{1}{|c|}{ Varones } & \multicolumn{1}{|c|}{ Mujeres } \\
\hline 1er. lugar & $\begin{array}{l}\text { Me siento muy bien pensando que } \\
\text { mi trabajo profesional será } \\
\text { ayudando al prójimo en la solución } \\
\text { de algún tipo de problema }\end{array}$ & $\begin{array}{l}\text { Me siento bien pensando que mi } \\
\text { trabajo profesional será ayudando } \\
\text { al prójimo en la solución de algún } \\
\text { tipo de problema }\end{array}$ \\
\hline 2do.lugar & $\begin{array}{l}\text { Me siento muy bien cuando pienso } \\
\text { que mi trabajo como profesional } \\
\text { será proyectando líneas de } \\
\text { comercialización o de negocios }\end{array}$ & $\begin{array}{l}\text { Me siento muy bien cuando pienso } \\
\text { que mi trabajo proyectando líneas } \\
\text { de comercialización. }\end{array}$ \\
\hline 3er. lugar & $\begin{array}{l}\text { Me siento muy bien cuando pienso } \\
\text { que mi trabajo como profesional } \\
\text { será desarrollando proyectos para } \\
\text { la producción industrial }\end{array}$ & $\begin{array}{l}\text { Me siento muy bien cuando pienso } \\
\text { que mi trabajo como profesional lo } \\
\text { realizaré en espacios abiertos como } \\
\text { el aire libre, en el campo, en ruinas, } \\
\text { etc. }\end{array}$ \\
\hline 4to. lugar & $\begin{array}{l}\text { Me siento muy bien cuando pienso } \\
\text { que mi trabajo como profesional lo } \\
\text { realizaré en espacios cerrados, } \\
\text { como oficinas, laboratorios, } \\
\text { consultorios. }\end{array}$ & $\begin{array}{l}\text { Me siento muy bien cuando pienso } \\
\text { que trabajo como profesional } \\
\text { será desarrollando proyectos para la } \\
\text { producción industrial }\end{array}$ \\
\hline 5to. lugar & $\begin{array}{l}\text { Me siento muy bien cuando pienso } \\
\text { que mi trabajo como profesional lo } \\
\text { realizaré en espacios abiertos como } \\
\text { el aire libre, en el campo, en } \\
\text { ruinas, etc. }\end{array}$ & $\begin{array}{l}\text { Me siento muy bien cuando pienso } \\
\text { que mi trabajo como profesional lo } \\
\text { realizaré en espacios cerrados, } \\
\text { como oficinas, laboratorios, } \\
\text { consultorios. }\end{array}$ \\
\hline
\end{tabular}

De la lectura de las últimas tablas se deduce que las percepciones, están asociadas a contenido autorrealizantes cuando afirman que así lograrán realizar sus metas y expectativas de vida así mismo están asociados a contenidos estóicos, cuando afirman que estudian la profesión como un reto por lo difícil que es.

Por otro lado la aptitud que se autoperciben poseer corresponde a la facilidad para el cálculo y la interpretación de textos científicos.

Como característica de su personalidad se perciben con la necesidad de que reconozcan su esfuerzo para continuar trabajando, y finalmente comunican sentirse muy cómodos al saber que su trabajo profesional será ayudando al prójimo a la solución de algún tipo de problema.

\section{DISCUSIÓN}

Según el enfoque evolutivo en la asesoría vocacional, se afirma que el joven entre los 18 a 24 años se encuentra en la etapa conflictiva entre optar por una profesión u ocupación que lo reafirme como persona o por lo contrario dedicarse a alguna ocupación que le permita a corto plazo acceder al mundo laboral, es entonces una etapa de reafirmación, punto que se encuentra en el presente estudio, donde las acciones basados en el gran esfuerzo y sacrificio tienen un especial significado y más aún cuando les permite conectarse con la posibilidad de ayudar al prójimo.

Por otro lado el papel de género para acceder a algunas profesiones, continua extinguiéndose, de forma tal que los varones no difieren de las mujeres en los conflictos vocacionales, ni en las percepciones ni expectativas. En el estilo atribucional, podríamos 
esperar que los sujetos que pretenden cambiarse de especialidad tenderán a un estilo atribucional pesimista, bajo el supuesto que postulan a una especialidad solo para asegurarse el ingreso. No obstante el resultado empírico nos dice que ambos grupos de estudiantes no difieren en cuanto al estilo atribucional y que ambos se encuentran en un nivel de búsqueda de indicadores que les dé motivo a ser optimistas o pesimistas, esto en cuanto se ubican en el grado cuatro de una escala de siete, pero es un cuatro con tendencia al cinco, es decir hacia la internalidad, lo que significa que el sujeto tiende a autoculparse por todo lo malo que le puede ocurrir, afortunadamente es contrarrestado por la especificidad y la inestabilidad, estos dos componentes ubican a nuestros jóvenes con tendencia al pesimismo, sin embargo puede revertirse, repetimos porque no generalizan lo malo ni son resonantes en el sentido de considerar permanente las situaciones penosas. En suma al no encontrar diferencias significativas entre los estudiantes que ingresaron a la universidad pensando en cambiarse a otra especialidad no los hace diferentes en las expectativas ambos se mueven por la vocación y la autorealización, las percepciones de sus habilidades, personalidad y criterios ocupacionales tampoco difieren, lo que significa que ambos grupos se orientan hacia metas similares, con estrategias diferentes. Tampoco aquí el sexo plantea diferencias lo que indica que el problema de género está dejando de ser una limitación en nuestro medio, y contrariamente a lo que se podría pensar que algunas facultades son percibidas como trampolín, no es verdad puesto que en ninguna de las escuelas académico profesionales estudiadas se observa porcentajes predominantemente significativos de jóvenes que desean cambiarse la tendencia es uniforme en cuotas cercanas al $12 \%$.

\section{CONCLUSIONES}

1. Se acepta la hipótesis alterna uno, por la que se puede afirma que los estudiantes que expresan su deseo de mantenerse hasta concluir sus estudios en la especialidad a la que ingresaron difieren en sus expectativas de sus pares que expresan su deseo de cambiarse de Escuela Académico Profesional.

2. Se acepta la hipótesis alterna dos afirmando que los estudiantes que expresan su deseo de mantenerse hasta concluir sus estudios en la especialidad a la que ingresaron difieren en sus percepciones hacia la profesión elegida respecto a los estudiantes que expresan su deseo de cambiarse de Escuela Académico Profesional, siendo la percepción favorable hacia la especialidad de ingreso, en los estudiantes que no presentan intención de traslado .

3. Se rechaza la hipótesis alterna tres, ya que, los estudiantes que expresan su deseo de cambiarse de Escuela Académico Profesional no difieren en sus estilos atribucionales con respecto a las causas que determinaron su ingreso a la universidad respecto a los estudiantes que expresan su deseo de mantenerse hasta concluir sus estudios en la especialidad a la que ingresaron.

4. Se rechaza la hipótesis alterna cuatro, al no encontrar que en función al sexo las expectativas de los ingresantes. que desean traslado de especialidad difieran de quienes manifiestan su permanencia.

Así mismo las percepciones hacia la especialidad de ingreso tampoco son diferentes entre los ingresantes que expresan su deseo de traslado de quienes expresan su deseo de permanecer en ella.

En lo que respecta al estilo atribucional, tanto quienes expresan su deseo de traslado como los que expresan su deseo de permanecer en la especialidad profesional a la que ingresaron tienden al estilo de causalidad atribucional optimista.

5. Se rechaza la hipótesis alterna cinco, al no encontrar: asociación significativa entre la Escuela Académico a la que se ingresó con sus expectativas y percepciones en función a 
su deseo de traslado o permanencia.

Así mismo respecto al estilo atribucional y la Escuela Académico Profesional no se ha encontrado en ningún caso un valor Ji cuadrado significativo, afirmando que el estilo atribucional optimista o pesimista no es característico de una determinada Escuela Académico Profesional ni tampoco de los estudiantes que expresan su deseo de o no trasladarse a otra especialidad profesional.

6. Al encontrar en el estilo atribucional, tendencia a la internalidad, siendo significativamente mayor en las mujeres, es esta dimensión la que requiere de trabajo urgente, puesto que puede llevar hacia la baja autoestima, la frustración y desmoralización, sin contar la tendencia a la depresión y trastornos psicosomáticos.

\section{BIBLIOGRAFIA}

Casulla M., Cayssials. Proyecto de vida y decisión vocacional. Paidos. B. Aires. 1994

Hamlyn, D.W. Experiencia y desarrollo del entedimiento. Herder. Barcelona 1981.

Madsen, K.B. Teorías de la Motivación. Paidos. Buenos Aires. 1967.

OIT Informe sobre el empleo en el mundo 1998-1999. Papel fundamental de la formación.

Peterson y Villanova. An expanded Atributional Style Questionaire. Journal Of Abnormal Psycholoy, 91,87.

Rivas Francisco. Psicología Vocacional: enfoques del asesoramiento. Morata. Madrid 1988.

Seligman Hellesner: On depressión, development and death. San Francisco B.H. Freeman.

Titze, M. Fundamentos del teleonálisis adleriano. Barcelona. Ed. Herder. 1983. 